\title{
PLATELET RICH PLASMA IN ANDROGENIC ALOPECIA IN MALE AND FEMALE PATIENTS- A MYTH, A PROSPECTIVE STUDY
}

\author{
S. Jhansi Lakshmi ${ }^{1}$, Dhanya Sri², J. Rupa Ramani ${ }^{3}$, Shaik Asha ${ }^{4}$, Naveen Kumar 5 , Sameera ${ }^{6}$, Amrutha Bindhu' ${ }^{7}$ Hanusha $^{8}$
}

${ }_{1}^{1}$ Assistant Professor, Department of DVL, Andhra Medical College, KGH, Visakhapatnam, Andhra Pradesh. ${ }^{2}$ Assistant Professor, Department of DVL, Andhra Medical College, KGH, Visakhapatnam, Andhra Pradesh. ${ }^{3}$ Postgraduate Student, Department of DVL, Andhra Medical College, KGH, Visakhapatnam, Andhra Pradesh. 4Postgraduate Student, Department of DVL, Andhra Medical College, KGH, Visakhapatnam, Andhra Pradesh. ${ }^{5}$ Postgraduate Student, Department of DVL, Andhra Medical College, KGH, Visakhapatnam, Andhra Pradesh. ${ }_{6}^{6}$ Postgraduate Student, Department of DVL, Andhra Medical College, KGH, Visakhapatnam, Andhra Pradesh. ${ }^{7}$ Postgraduate Student, Department of DVL, Andhra Medical College, KGH, Visakhapatnam, Andhra Pradesh. ${ }^{8}$ Postgraduate Student, Department of DVL, Andhra Medical College, KGH, Visakhapatnam, Andhra Pradesh.

\section{ABSTRACT}

\section{BACKGROUND}

Androgenetic alopecia (AGA) is a hereditary, androgen-dependent dermatological disorder more common in men. It is occasionally seen in women. It commonly begins by 20 years of age and affects nearly $50 \%$ of men by the age of 50 years. It is a progressive thinning of the scalp hair in a defined pattern causing significant lowering of the self-esteem and psychological well-being of the patient. The treatment modalities are limited, mainly minoxidil, 5-alpha-reductase inhibitors and hair transplantation. Few treatment options and those too having side effects prompted the discovery of platelet-rich plasma (PRP).(1) The basic idea behind PRP injection is to deliver high concentrations of growth factors to the scalp with the hope of stimulating hair regrowth. PRP is an autologous preparation of platelets in concentrated plasma. Although, the optimal PRP platelet concentration is unclear, the current method by which the PRP is prepared reports $300-700 \%$ enrichment with platelets concentration consequently increasing to more than 1,000,000 platelets. PRP has attracted attention in several medical fields, because of its ability to promote wound healing.(2) Activation of alpha granules of platelets releases numerous proteins including platelet derived growth factor (PDGF), transforming growth factor (TGF), vascular endothelial growth factor (VEGF) and interleukin (IL-1). It is hypothesised that growth factors released from platelets may act as a stem cell in the bulge area of the follicles stimulating the development of new follicles and promoting neovascularisation.

Aims and Objectives- This is uncontrolled clinical trial done to know the safety, efficacy and feasibility of PRP injections in treating androgenic alopecia in both male and female patterns hair loss.

\section{MATERIALS AND METHODS}

This study was uncontrolled clinical trial. A total of 32 patients were selected, out of which 18 patients suffering from hair loss due to androgenic alopecia (56\%) and 14 cases of female pattern hair loss (44\%) not responding to 6 months treatment with minoxidil and finasteride were included in the study. A total volume of 2 to $3 \mathrm{cc}$ of PRP was injected in the scalp using an insulin syringe. The treatment was repeated every 2 weeks for a total of 4 times. The outcome was accessed after 3 months by clinical examination, by taking photos and doing hair pull test.

Study Setting- DVL Department, Andhra Medical College, King George Hospital, Visakhapatnam, Andhra Pradesh, India.

\section{RESULTS}

A significant reduction of hair loss was observed between $1^{\text {st }}$ and $4^{\text {th }}$ injection. There was significant improvement in the hair growth after $6^{\text {th }}$ injection in 22 patients and hair pull test became negative in 13 of the above cases.

\section{CONCLUSION}

PRP is a single, cost effective and feasible treatment option for androgenic alopecia. In both male and female patterns, it showed good results.

\section{KEY WORDS}

Androgenic Alopecia, Male and Female Pattern, Platelet Rich Plasma, Hair Pull Test.

HOW TO CITE THIS ARTICLE: Lakshmi SJ, Sri D, Ramani JR, et al. Platelet rich plasma in androgenic alopecia in male and female patients- a myth, a prospective study. J. Evolution Med. Dent. Sci. 2018;7(21):2522-2526, DOI: 10.14260/jemds/2018/567

'Financial or Other Competing Interest': None.

Submission 02-12-2017, Peer Review 04-05-2018,

Acceptance 11-05-2018, Published 21-05-2018.

Corresponding Author:

Dr. Dhanya Sri,

Assistant Professor, DVL, KGH, AMC,

Maharanipeta, Visakhapatnam-530002,

Andhra Pradesh.

E-mail: ashashaik72@gmail.com

DOI: $10.14260 /$ jemds $/ 2018 / 567$

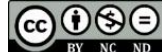

\section{BACKGROUND}

Androgenic alopecia, a male pattern baldness is very common type of hair loss observed in both male and female. PRP is an autologous preparation of platelets in concentrated plasma. Although, the optimal PRP platelet concentration is unclear, the current method by which the PRP is prepared reports 300 - 700\% enrichment with platelets concentration consequently increasing to more than $1,000,000$ platelets. PRP has attracted attention in several medical fields, because of its ability to promote wound healing. Activation of alpha granules of platelets releases numerous proteins including platelet 
derived growth factor (PDGF), transforming growth factor (TGF), vascular endothelial growth factor (VEGF) and interleukin (IL-1). It is hypothesised that growth factors released from platelets may act as a stem cell in the bulge area of the follicles stimulating the development of new follicles and promoting neovascularisation.

The aim of our study was to evaluate the safety, efficacy and feasibility of PRP for treatment of androgenic alopecia in both male and females by staging the patient based on Norwood-Hamilton scale and Ludwig classification respectively.

\section{Norwood Hamilton Scale- Stage 1}

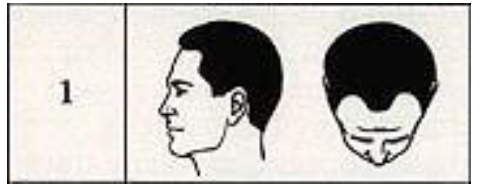

Very minor or no recession of the hair line, therefore no need for treatment. Unless you have a family history of baldness, there is no need to worry. If there is a family history of male baldness, you may want to monitor the situation closely and decide the appropriate time for treatment.

\section{Norwood-Hamilton Scale- Stage 2}

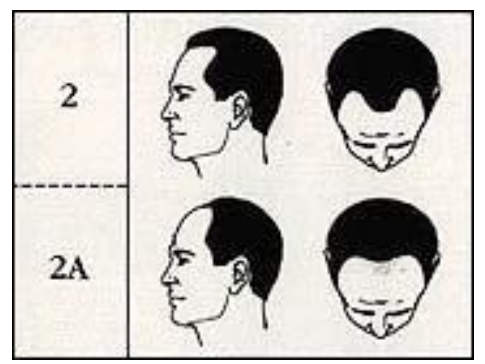

Triangular and typically symmetrical areas of recession at the front temporal area. Hair loss remains ahead of a line several centimetres in front of the ears. Hair falls and may become less dense in the central front part of the scalp. Initial signs of baldness are becoming evident.

Norwood-Hamilton Scale- Stage 3

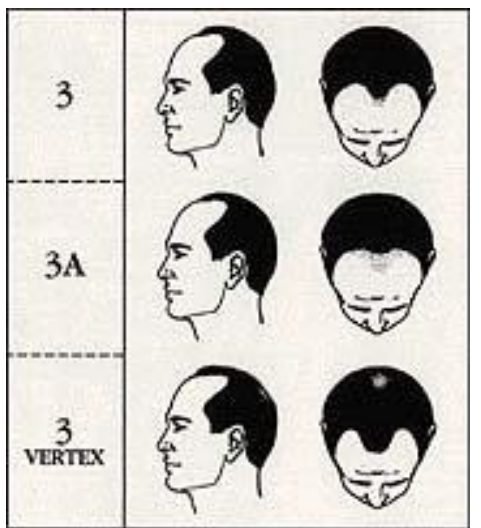

This represents the lowest extent of hair loss considered sufficient to be called baldness according to Norwood. Most scalps at this stage have deep symmetrical recession showing at the temples that are bare or only sparsely covered by hair. With stage 3 vertex, the crown is added since it is a common occurrence with age. Hair loss is primarily from the vertex with limited recession of the front temporal hairline.

\section{Norwood-Hamilton Scale- Stage 4}

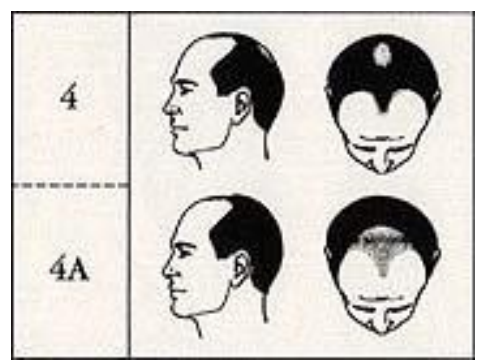

Recession at the front temporal areas is more severe than stage 3. There is a decisive lack of hair on the crown. A band of moderately dense hair extending across the top separates the two areas of hair loss between front temporal and crown. This band bridges between the hair covered areas on the side of the head.

\section{Norwood-Hamilton Scale- Stage 5}

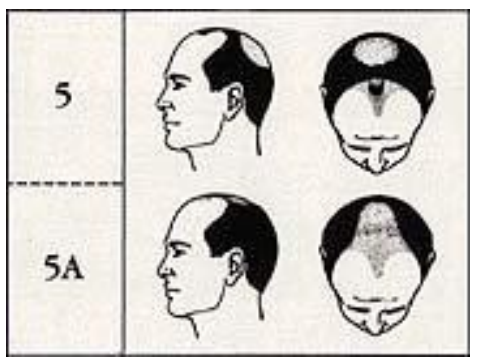

At stage 5 hair loss at the vertex region is still separated from the front temporal region, but the division is much less distinct. The band of hair extending across the crown is noticeably narrower and thinner. Hair loss at the vertex and front temporal regions are larger. When viewed from above, stages 5 to 7 show the remaining hair at the sides and back as a distinct horseshoe shape.

Norwood-Hamilton Scale- Stage 6

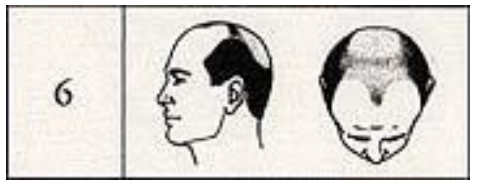

The bridge of hair that once crossed the crown has now been lost with only sparse hair remaining. The front temporal and vertex regions are now joined into one area. Hair loss on the sides has extended further.

Norwood-Hamilton Scale- Stage 7

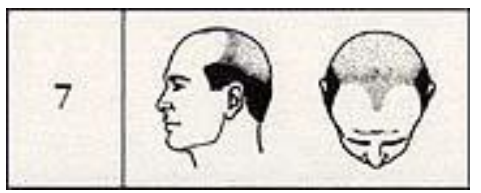


This is the most advanced or severe form of hair loss. Only a narrow band of hair in a horseshoe shape survives on the sides and back of the scalp. This hair may be fine and less dense than before. At the nape of the neck, the hair is sparse with a semicircle over both ears.

\section{The Ludwig Scale}

The Ludwig Scale uses 3 different classifications or Types to diagnose the severity of female hair loss. From left to right in the image below, these types include Type I, Type II and Type III.

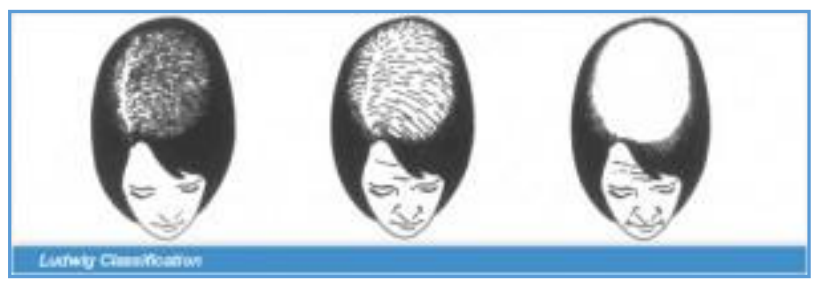

\section{Type I}

In this stage, hair loss is considered to be mild. Most women may have difficulty noticing that hair loss has occurred, as the frontal hairline remains relatively unaffected. Hair loss may occur on the top and front of the scalp, however. Such hair loss may be noticeable when the hair is parted down the centre of the scalp, as more and more scalp will become visible over time.

\section{Type II}

Type II hair loss is considered moderate. In this stage, women may notice each of the following: Thinning, shedding, general decrease in volume and a centre part that continues to widen over time. Depending on the severity, a hair transplant procedure may be a viable option for women who exhibit a Type II classification.

\section{Type III}

Type III is the final and most extreme classification of female hair loss. In this stage hair is so thin that it has difficulty camouflaging the scalp, rendering it visible to the naked eye. This may be worsened by a number of factors including hair miniaturisation, progressive thinning and extensive loss.

\section{MATERIALS AND METHODS}

It is uncontrolled clinical trial. The study was done in July 2014 and June 2015; all the patients suffering from androgenic alopecia and topical minoxidil and finasteride for at least 6 months without much improvement were considered for PRP therapy. Written informed consent was obtained. All included patients were tested for ELISA, for HIV, HBsAg and platelet count. Exclusion criteria were haematological disorders, thyroid dysfunction, malnutrition and other dermatological disorders contributing to hair loss. Sample size was taken conveniently.

A $1 \mathrm{~cm}$ to $1 \mathrm{~cm}$ square area was marked over right parietal area in the mid-pupillary line, $10 \mathrm{~cm}$ proximal to right eyebrow in each patient. Baseline follicular unit were manually counted with the help of dermascope.

\section{Patient Evaluation}

Before each session, the hair pull test was performed three times by the same clinician. All patients were advised to avoid washing hair 2 days prior to treatment. A bundle of approximately 50 to 60 hairs were grasped with thumb and index and middle finger from base close to the scalp. The hair was counted in each session. To evaluate overall hair growth, hair volume, hair quantity and fullness, pictures were taken in each session from front, vertex, lateral and back view.

PRP was prepared by collecting $20 \mathrm{cc}$ of fresh blood in sodium citrate containing vacutainers in minor operation theatre under proper aseptic precautions. The tubes were rotated in a centrifuge machine 1500 to $2000 \mathrm{rpm}$, that is "soft spins" which allows blood separation into 3 layers, bottom RBC layer ( $55 \%$ of total volume), top most acellular plasma layer called platelet poor plasma (PPP) and $40 \%$ of total volume and an intermediate PRP layer of $5 \%$ of total volume called the "Buffy Coat." Separated Buffy coat with PPP was collected with the help of fine pipette in another test tube. This tube underwent a second centrifuge, which was longer and faster than the $1^{\text {st }}$ called "hard" spin comprising $2500 \mathrm{rpm}$ for 15 mins. This allows the PRP to settle at the bottom of the tube. The upper layer containing PPP was discarded and the lower layer of PRP was loaded in an insulin syringe containing calcium chloride (1 part calcium chloride and 9 parts of PRP) as an activator.

One hour prior to administration, PRP anaesthetic cream was applied over the bald area. Area of the scalp to be treated was cleaned with spirit and povidone-iodine. With the help of insulin syringe, PRP was injected over affected area by multiple small injections in a linear pattern $1 \mathrm{~cm}$ apart called (Nappage) technique under proper aseptic condition in minor operation theatre. A total volume of 2 to $3 \mathrm{cc}$ was injected. The treatment was repeated for every 2 to 3 weeks for 4 sessions. At each visit, hair counts were noted. Subjective improvements of patients were noted. We evaluated all the patients at the end of 12 weeks.

\section{Objectives of the Study}

It is uncontrolled clinical trial done to know the safety, efficacy and feasibility of PRP injections in treating androgenic alopecia in both male and female patterns hair loss.(3)

\section{Study Setting}

DVL Department, Andhra Medical College, King George Hospital, Visakhapatnam, Andhra Pradesh, India.

\section{Statistical Analysis}

Data was analysed using SPSS Version 16.0. The Qualitative Data were expressed as numbers and percentages.

\section{RESULTS}

16 male patients in the age group of 25 to 50 years were included in the study. According to Hamilton classification of male pattern baldness 6 patients were in grade 2, 5 patients were in grade 3 and 4 patients were in grade 4 androgenic alopecia. Female pattern was also graded in 14 patients.

Before treatment, all our patients has a positive hair pull test in a mean number of 10 hairs. After $4^{\text {th }}$ session, hair pull test was negative in 9 patients with an average number of three hairs. A significant reduction in hair loss was observed 
between $1^{\text {st }}$ and $4^{\text {th }}$ injections as noticed by patients. Global pictures also revealed a moderate improvement in hair volume and coverage in 23 patients out of 30 patients.

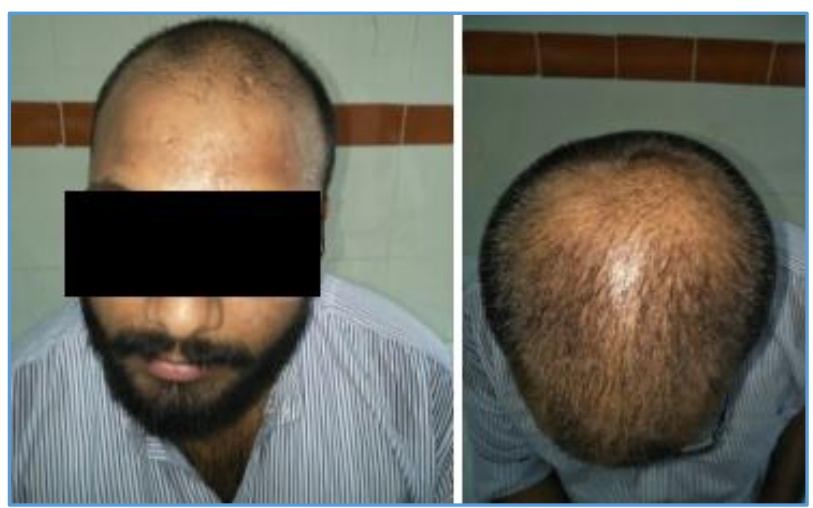

Male Pattern Baldness before PRP

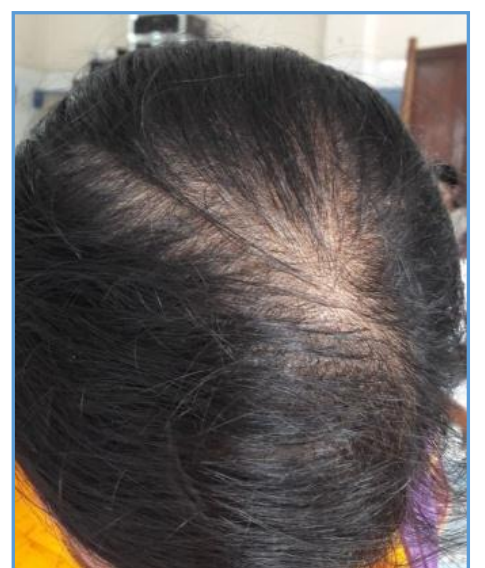

Female Pattern Baldness before PRP

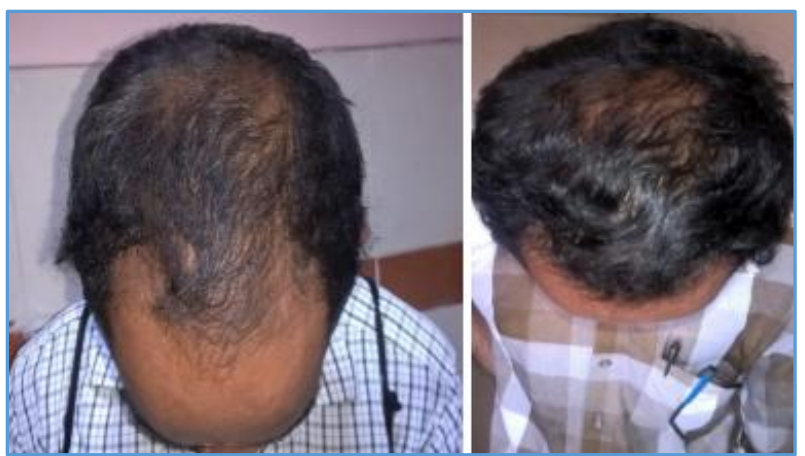

Male Pattern Baldness after PRP

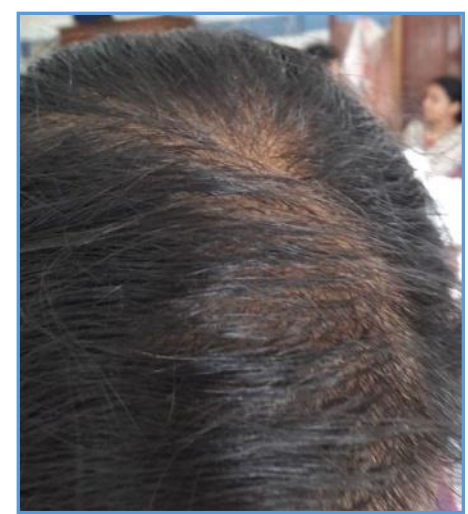

Female Pattern Baldness after PRP
Side effects after PRP injections were minimal pain, redness at the time of injection and pin point bleeding was observed.

\section{DISCUSSION}

Hair loss has a significant influence on psychological distress and is associated with low self-esteem and depression. Treatment options for androgenic alopecia are very limited and include topical minoxidil and oral finasteride, either alone or in combination. $(4,5,6,7)$ However, there are several reported side effects such as headache and increased body hairs for minoxidil, whereas loss of libido has been reported with oral finasteride. Finasteride also interferes with genital development in a male foetus and is contraindicated in a pregnant woman and those likely to become pregnant.

PRP has already attracted attention in others like plastic surgery. Growth factors are known to activate the proliferative phase and trans-differentiation of hair and stem cells to produce new follicular units. VEGF is reported to promote in vitro proliferation of papilla cells and thereby plays a key role in elongating hair shaft.(8)

We prepared PRP by double spin method, in which blood cell layers were normally separated; activation of platelets through coagulation triggers the secretion of various growth factors which produce mitogenic effects in various cell types. Activated PRP promotes the proliferation of dermal papillary cells and prevents their apoptosis.

In our study, the hair pull test became negative after 4 sessions of PRP. This finding is comparable with the study conducted by Besti et al. This study also observed significant improvement in hair volume and coverage in global pictures, but according to our study moderate improvement in hair volume and coverage was observed.

Uebel et al observed a significant improvement in hair density and stimulation of growth when follicular units were pre-treated with platelet plasma growth factors before the implantation. (9) There was significant difference in the yield of follicular unit on comparing with the experiments with the controlled areas of scalp.(10) The areas treated with platelet plasma growth factors demonstrated a yield of 18.7 follicular units per square centimetres, whereas the control area yield 16.4 follicular units per square $\mathrm{cm}$ and increase in follicular density by $15.1 \%$.

Our study has some limitations. Sample size is smaller. Mean follow-up of patients was also short to draw conclusion of long-term effective treatment.

\section{CONCLUSION}

PRP injections for androgenic alopecia was simple, cost effective and feasible treatment option for hair loss and can be regarded as a valuable treatment modality for androgenic alopecia.(11) Although, PRP has a sufficient theoretical and scientific basis to support its role in hair restoration. PRP is still in its infancy. Clinical evidence is still weak, considering its excellent safety profile and relatively low cost. PRP is a promising treatment option for patients with thinning of hair. 


\section{REFERENCES}

[1] Li ZJ, Choi HI, Choi DK, et al. Autologous platelet-rich plasma: a potential therapeutic tool for promoting hair growth. Dermatol Surg 2012;38(7 Pt 1):1040-6.

[2] Marx RE. Platelet-rich plasma: evidence to support its use. J Oral Maxillofac Surg 2004;62(4):489-96.

[3] Uebel CO, da Silva JB, Cantarelli D, et al. The role of platelet plasma growth factors in male pattern baldness surgery. Plast Reconstr Surg 2006;118(6):1458-66.

[4] Raja SV, Naidu ME. Platelet-rich fibrin: evolution of a second-generation platelet concentrate. Indian J Dent Res 2008;19(1):42-6.

[5] Olsen EA, Weiner MS, Delong ER, et al. Topical minoxidil in early male pattern baldness. J Am Acad Dermatol 1985;13(2 Pt 1):185-92.

[6] Kaufman KD, Olsen EA, Whiting D, et al. Finasteride in the treatment of men with androgenetic alopecia. Finasteride Male Pattern Hair Loss Study Group. J Am Acad of Dermatol 1998;39(4 Pt 1):578-89.
[7] Messenger AG. Medical management of male pattern hair loss. Int J Dermatol 2000;39(8):585-6.

[8] Gardner MJ, Demetrakopolous D, Klepchick PR, et al. The efficacy of autologous platelet gel in pain control and blood loss in total knee arthroplasty. An analysis of the haemoglobin, narcotic requirement and range of motion. Int Orthop 2007;31(3):309-13.

[9] Glover JL, Weingarten MS, Buchbinder DS, et al. A 4year outcome-based retrospective study of wound healing and limb salvage in patients with chronic wounds. Adv Wound Care 1997;10(1):33-8.

[10] Katsuoka K, Schell H, Wessel B, et al. Effects of epidermal growth factor, fibroblast growth factor, minoxidil and hydrocortisone on growth kinetics in human hair bulb papilla cells and root sheath fibroblasts cultured in vitro. Arch Dermatol Res 1987;279(4):247-50.

[11] Besti EE, Germain E, Kalbermatten DF, et al. Plateletrich plasma injection is effective and safe for the treatment of alopecia. Eur J Plast Surg 2013;36:407-12. 